\title{
Blood flow reactivity to hypercapnia in strictly unilateral carotid disease: preliminary results
}

\author{
Ross L Levine, Jeffrey A Dobkin, Jack M Rozental, Martin R Satter, Robert J Nickles
}

\begin{abstract}
To show a relationship between degree of carotid arterial stenosis and cerebral blood flow reactivity (RES\%) to induced hypercapnia, fluorine-18-fluoromethane and positron emission tomography (PET) was used to study 18 patients with carotid distribution transient ischaemic attacks (TIA), all free of stroke, who had angiographic-proven unilateral arterial disease. Non-involved carotid arteries were either normal or had non-stenotic plaque. Either normal arteries or nonstenotic ulcerations in the symptomatic carotid arteries were present in five of 18 (28\%), ipsilateral carotid stenosis from $50-99 \%$ was present in eight of $18(44 \%)$, and ipsilateral internal carotid occlusion was present in five of $18(28 \%)$ patients. In comparison with 14 normal controls, all patients with symptomatic middle cerebral artery (MCA) flow territories had significantly lower mean (SEM) RES\% [5.0 (0.2) vs 4.0 (0.9), p < 0.04].
\end{abstract} Symptomatic anterior borderzone (ABZ) RES\% was also significantly lower [4.6 $(0.4)$ vs $3.3(0.9), \mathrm{p}<0.04]$, than controls. In patient subgroup comparisons, the $50-99 \%$ stenosis subgroup clearly had the lowest MCA RES\% [3.4 $(0 \cdot 2)]$ as well as the lowest ABZ RES\% [2.8 $(0.4)]$ on their symptomatic sides. Age, expired $\mathrm{pCO}_{2}$, mean arterial blood pressure, serum glucose, serum haematocrit and number, type and estimated duration of TIAs were not significantly different between subgroups. Linear regression showed a significant relationship between RES\% and both measured percentage-stenosis $(p=0.04)$ and residual luminal diameter $(p=0.05)$ in symptomatic MCA territories. This approached significance in symptomatic ABZ regions. This preliminary data set suggests that unilateral carotid stenosis can and does result in impaired $\mathrm{CO}_{2}$ reactivity following hypercapnia. The relative normality of $\mathrm{CO}_{2}$ reactivity in those with carotid occlusion is discussed.

Stroke occurs as a phenomenon related to variable degrees of extracranial vascular occlusive disease but is dependent on several unpredictable factors such as dislodgement of arterial thrombi from an atherosclerotic plaque, cerebral blood flow (CBF) restriction secondary to haemodynamic changes associated with flow through a "critical" stenosis, thromboembolism precipitated by "subcritical" stenosis or secondary to haemodynamic changes associated with arterial lesions and the overall effectiveness of collateral blood flow. The assessment of these haemodynamic changes is paramount in further understanding the pathogenesis of occlusive vascular disease. ${ }^{1}$

In patients with transient ischaemic attacks (TIAs) and angiographically identified atherosclerotic lesions, Grotta et al found that carotid stenosis greater than $50 \%$ was statistically related to a higher incidence of ipsilateral stroke compared with those with carotid stenosis less than $50 \% .^{2}$ Clinicopathological findings suggest that a stenotic atherosclerotic lesion may progress to a more stenotic lesion or vessel occlusion, ${ }^{2-4}$ which may result in stroke or TIA. The "subcritical" nature of carotid artery stenosis is believed to be reached when vessel diameter is reduced by $60-65 \%$, producing a residual luminal diameter of $1-2 \mathrm{~mm} .{ }^{156}$ Significant pressure drops do not occur distal to a stenosis of less than $72 \%$ luminal area reduction; pressure decreases do occur if cross-sectional luminal area is less than $2 \mathrm{~mm}^{2}$ or with stenosis greater than $87 \%$ luminal area. ${ }^{47}$ The effects of percentage reductions between these yalues is unclear.

We have studied 18 patients who have strictly unilateral extracranial carotid arterial disease using positron emission tomography (PET), fluoromethane $\left({ }^{18} \mathrm{FCH}_{3}\right)$ and $\mathrm{CBF}$ reactivity to induced hypercapnia. In this preliminary study we aimed to non-invasively study the physiological effect of varying degrees of arterial stenosis on the cerebral circulation.

\section{Patients}

Among 53 consecutive TIA patients studied at the University of Wisconsin Clinical PET Center between 1983-87, 18 patients with unilaterality of both symptoms and carotid disease and no evidence of previous infarction were identified. All patients were examined by the Stroke Consultation Service (RLL, JAD) at the William $S$ Middleton Memorial Veterans Hospital or at the University of Wisconsin Hospital and Clinics. Data were collected prospectively.

All patients were examined neurologically and had CT, angiographic and PET studies between one and nine days of the last TIA. No patient had PET during or within 24 hours of TIA. By entrance criteria to this 
particular study, all plain CTs were normal. All selective angiograms were inspected (RLL or JAD) for arterial occlusive disease in the extracranial circulation. Patients were free of intracranial and contralateral disease other than non-stenotic plaque.

Examiners were blind to both clinical and PET information when they graded ipsilateral internal carotid artery (ICA) lesions. We used the methodology of Powers et $a l^{1}$ and measured the diameter of the ipsilateral ICA at its origin, the level of the first cervical vertebra (CI), and the siphon. Measurements were taken on both lateral and anteroposterior projections, and the mean value was determined as the luminal diameter at that site. The percentage stenosis for each patient was calculated from the luminal diameter at the site of stenosis and the diameter at CI. As necessary, measurements were corrected for magnification. Patients were separated into those with either normal or non-stenotic ICAs $(\mathrm{n}=5$, Group A), those with 50-99\% luminal stenosis ( $n=8$, Group B), and those with ICA occlusion ( $n=5$, Group $C)$. When an ICA occlusion was present, the source of collateral supply was noted. Groups B and C were dealt with as separate groups because potential surgical interventional therapy would be different for each group. We did not calculate residual luminal area at this time, ${ }^{7}$ but do intend to add this measurement when our data set is expanded into a larger number of subjects. At this point, we used the same measurements as Powers et al ${ }^{1}$ to enable us to compare results more directly.

Detailed accounts of the TIA number, type and duration were taken. One patient in each of Groups B and C had apparent haemodynamic induction of their TIAs. Thus we did not attempt to separate this preliminary data set into haemodynamic and non-haemodynamic subgroups as has been previously reported. ${ }^{8}$ Risk factor analysis was performed as obtained from patient history, chart review, or discussion with their primary care givers. Patients were not on central-acting sedatives or stimulants, anti-platelet agents other than aspirin, nor any of the drugs clearly recognised to alter CBF.

\section{Methods}

All dynamic PET data sets were collected and analysed blindly according to previously defined guidelines. ${ }^{89}$ Spatial resolution for the PET images was $16 \mathrm{~mm}$ in the plane of section; slice thickness was $18 \mathrm{~mm}$. All patients were studied at a single plane of study $5-6 \mathrm{~cm}$ above and parallel to the orbito-meatal line. Measured attenuation images were generated using a germanium- 68 source at the plane of study. A dynamic sequence of 12 one minute scans was initiated at inhalation of $25-40 \mathrm{mCi}$ ${ }^{18} \mathrm{FCH}_{3}$, which, with the measured expiredbreath activity curve, constituted input data for the derivation of best-fit CBF and blood-brain partition coefficients. The rapid biological clearance of the non-metabolised ${ }^{18} \mathrm{FCH}_{3}$ allowed serial normocapnic and hypercapnic examinations at 20 minute intervals with less than $5 \%$ of the activity administered persisting at that time. Induced hypercapnia consisted of inhalation of $5 \% \mathrm{CO}_{2}$ and $95 \% \mathrm{O}_{2}$.

Expired carbon dioxide $\left(\mathrm{PeCO}_{2}\right)$, mean arterial blood pressure (MABP), serum haematocrit and serum glucose values were measured during scanning sequences. All PET studies were performed after informed consent and were initiated after the subject had been quietly lying supine for at least 30 minutes. A concurrently seen group of neurologically normal, paid volunteers $(n=14)$ was chosen from our PET data bank for comparisons.

We defined middle cerebral artery (MCA), anterior borderzone (ABZ), and posterior borderzone (PBZ) flow territories for each determined RES\%, defined as the percentage change in CBF over baseline per mm $\mathrm{PeCO}_{2}$, and RES\% symmetry ratios, defined as symptomatic RES\% divided by asymptomatic RES\%, were recorded according to previous guidelines. ${ }^{8-10}$ Values were generated for both symptomatic and asymptomatic sides in a fashion modelled on the work of von Schulthees et $a l^{11}$ and Leblanc et al. ${ }^{12}$ The patient data chosen for this report represents a subset of previously published CBF data, ${ }^{89}$ but were selected to evaluate only those subjects with unilateral carotid disease.

Means and standard error of each measurement (SEM) as $t$ approximated the

Table 1 Epidemiological and clinical features

\begin{tabular}{lccc}
\hline & Group $A$ & Group B & Group C \\
\hline Number of subjects & 5 & 8 & 5 \\
Age range, years & $60-76$ & $48-76$ & $49-76$ \\
Mean age (SEM) & $65 \cdot 8(5 \cdot 5)$ & $59 \cdot 6(5 \cdot 7)$ & $64 \cdot 0(8 \cdot 7)$ \\
Number risk factors & $2 \cdot 0(1 \cdot 2)$ & $2 \cdot 4(0 \cdot 8)$ & $2 \cdot 0(1 \cdot 0)$ \\
Number of TIAs & & & $5 \cdot 4(5 \cdot 0)$ \\
$\quad$ Mean (SEM) & $4 \cdot 2(5 \cdot 3)$ & $5 \cdot 6(3 \cdot 5)$ & 3 \\
$\quad$ Median & 2 & $1-20$ & $1-20$ \\
$\quad$ Range & $1-15$ & 15 & 18 \\
Duration (min) & 10 & $1-600$ & $5-180$ \\
$\quad$ Median & $5-30$ & & $28 \%$ \\
$\quad$ Range & $20 \%$ & $30 \%$ & $43 \%$ \\
Symptoms & $60 \%$ & $40 \%$ & $43 \%$ \\
$\quad$ Amaurosis fugax & $60 \%$ & $40 \%$ & $14 \%$ \\
Motor loss & $20 \%$ & $10 \%$ & \\
Sensory loss & & & \\
Speech dysfunction & & & \\
\hline
\end{tabular}

Group A, non-stenotic ICA.

Group B, 50-99\% ICA stenosis

Group C, ICA occlusion. 
Table 2 Blood flow detail*

\begin{tabular}{|c|c|c|c|}
\hline & Group $A$ & Group B & Group $C$ \\
\hline $\begin{array}{l}\text { RES\%-MCA } \% \\
\text { Symptomatic side } \\
\text { Asymptomatic side } \\
\text { Ratio }\end{array}$ & $\begin{array}{l}4.7(0.5) \\
4.7(0.6) \\
1.0(0 \cdot 10)\end{array}$ & $\begin{array}{l}3.4(0.2) \\
3.5(0.2) \\
0.99(0.08)\end{array}$ & $\begin{array}{l}4.5(1.0) \\
5 \cdot 2(0.8) \\
0.86(0.16)\end{array}$ \\
\hline $\begin{array}{l}\text { RES\%-ABZ } \ddagger \\
\text { Symptomatic side } \\
\text { Asymptomatic side } \\
\text { Ratio }\end{array}$ & $\begin{array}{l}4.0(0.8) \\
4.6(0.8) \\
0.85(0.08)\end{array}$ & $\begin{array}{l}2.8(0.4) \\
3.8(0.4) \\
0.75(0.12)\end{array}$ & $\begin{array}{l}3.3(0.8) \\
4 \cdot 1(1.0) \\
0.81(0.08)\end{array}$ \\
\hline $\begin{array}{l}\text { RES\%-PBZ } \\
\text { Symptomatic side } \\
\text { Asymptomatic side } \\
\text { Ratio }\end{array}$ & $\begin{array}{l}4.4(1.0) \\
4.6(1.0) \\
0.96(0.08)\end{array}$ & $\begin{array}{l}3.4(0.2) \\
3.6(0.4) \\
0.94(0.06)\end{array}$ & $\begin{array}{l}4.4(1.4) \\
4.4(1.0) \\
0.99(0.14)\end{array}$ \\
\hline $\begin{array}{l}\mathrm{PeCO}_{2} \text { difference } \\
\mathrm{MABP} \\
\text { Serum haematocrit } \\
\text { Serum glucose } \\
\text { Number days between TIA and PET }\end{array}$ & $\begin{array}{c}11 \cdot 8(2 \cdot 2) \\
103 \cdot 8(7 \cdot 3) \\
44 \cdot 2(4 \cdot 2) \\
137 \cdot 4(26 \cdot 9) \\
4 \cdot 4(2 \cdot 7)\end{array}$ & $\begin{array}{c}11 \cdot 7(2 \cdot 6) \\
103 \cdot 1(6 \cdot 6) \\
44 \cdot 7(2 \cdot 0) \\
127 \cdot 6(21 \cdot 8) \\
3 \cdot 6(1 \cdot 5)\end{array}$ & $\begin{array}{c}13 \cdot 2(4 \cdot 9) \\
100 \cdot 2(3 \cdot 6) \\
45 \cdot 6(2 \cdot 7) \\
124 \cdot 0(21 \cdot 8) \\
3 \cdot 4(2 \cdot 0)\end{array}$ \\
\hline
\end{tabular}

^Expressed as mean (SEM) as $t$ approximates $95 \%$ limit.

+ Group $B$ values were significantly lower than other patient groups $(p<0.04)$

†roup $B$ values were significantly the lowest $(p<0.04)$ and all patient side to side ratios significantly lower than controls $(p<0.04)$.
Figure 1 Individual $M C A$ reactivity values for each subject along with group means (SEM). Symptomatic $\left({ }^{\star}\right)$ and asymptomatic MCA values are depicted. Between patient group comparisons show all Group $B$ values to be significantly lowered ( $p<$ $0.04)$.
95\% significance level were calculated. Nonparametric Kruskal-Wallis analysis of variance was used for between group comparisons. All patient RES\% values were first compared with normative data and then comparisons were made between patient groups. The Wilcoxon signed rank test was used to compare symptomatic versus asymptomatic measures for each group. We studied both RES\% and RES\% symmetry ratios. When multiple comparisons were made, Bonferroni's method of correction was employed. Linear regression was used to compare RES\% to both percentage ICA stenosis and residual ICA lumen.

\section{Results}

Epidemiological and clinical features (table 1) Although not strictly matched for age, the control and patient groups were comparable in terms of mean age, age range and both number and type of cerebrovascular risk factors. Subjects were free of diabetes mellitus, renovascular hypertension and difficult to control hypertension. We did not measure chronicity of hypertension per se. Although not statistically different, Group B had more TIAs per patient while Group C had slightly longer TIA duration as reported subjectively. Symptom

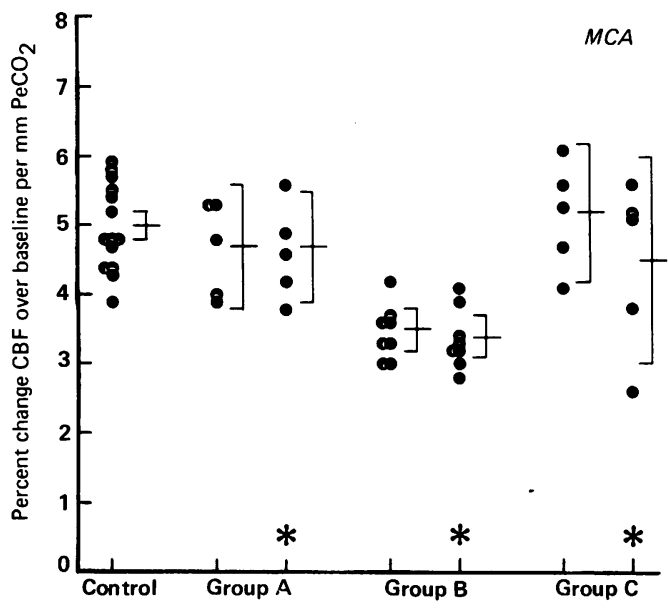

Table 3 Normative data ${ }^{\star}$

\begin{tabular}{ll}
\hline & Control population \\
\hline Number of subjects & 14 \\
Age range, years & $23-78$ \\
Mean age (SEM) & $57 \cdot 8(10 \cdot 2)$ \\
CBF values $\dagger$ & \\
Mean MCA RES\% & $5 \cdot 0(0 \cdot 2)$ \\
95\% confidence interval & $3 \cdot 8(6 \cdot 2)$ \\
L/R ratio & $1 \cdot 01(0 \cdot 04)$ \\
Mean ABZ RES\% & $4 \cdot 6(0 \cdot 4)$ \\
95\% confidence interval & $2 \cdot 5(6 \cdot 7)$ \\
L/R ratio & $1 \cdot 02(0 \cdot 04)$ \\
Mean PBZ RES\% & $4 \cdot 1(0 \cdot 27)$ \\
PeCO difference & $11 \cdot 5(1 \cdot 6)$ \\
\hline
\end{tabular}

$\star$ Expressed as mean (SEM) as $t$ approximates the $95 \%$ limit. †Mean value is mean of left plus right side.

breakdown was also comparable between groups.

Blood flow detail-MCA (table 2) Graphically (fig 1) and statistically, the MCA RES\% of Group B was clearly the lowest in comparisons made between patient groups $(p<0.04)$. Control normative data (table 3 ) were generated and used in graphic comparisons. $\mathrm{PeCO}_{2}$ differences were comparable between controls and patient groups. In addition, MABP, serum haematocrit and serum glucose values on the day of CBF study were comparable between patient groups. Side to side ratios were not significantly different for either controls versus patients (fig 2) or between patient groups. Symptomatic versus asymptomatic RES\% values were surprisingly not significantly different within each respective group, but the difference between symptomatic and asymptomatic Group C regions does approach significance. Of importance, is the fact that four out of five Group $C$ patients stopped having TIAs by the time CBF studies were completed. The fifth patient, with a symptomatic MCA RES \% of $2 \cdot 6$, appeared to have both haemodynamic induction of TIAs and continued events.

Blood flow detail-ABZ (table 2) Graphically

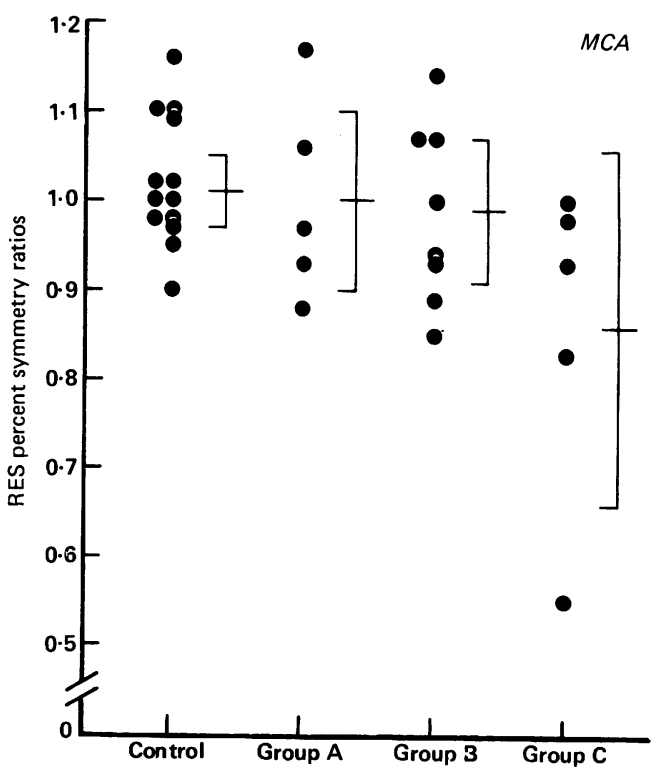

Figure 2 Individual $M C A$ ratios for each subject with group means (SEM). Ratios are not significantly different between groups. 
Figure 3 Individual $A B Z$ reactivity values for each subject along with group means (SEM). Between patient group comparisons show that Group B symptomatic values $(\star)$ are significantly lowered $(p<0.04)$.
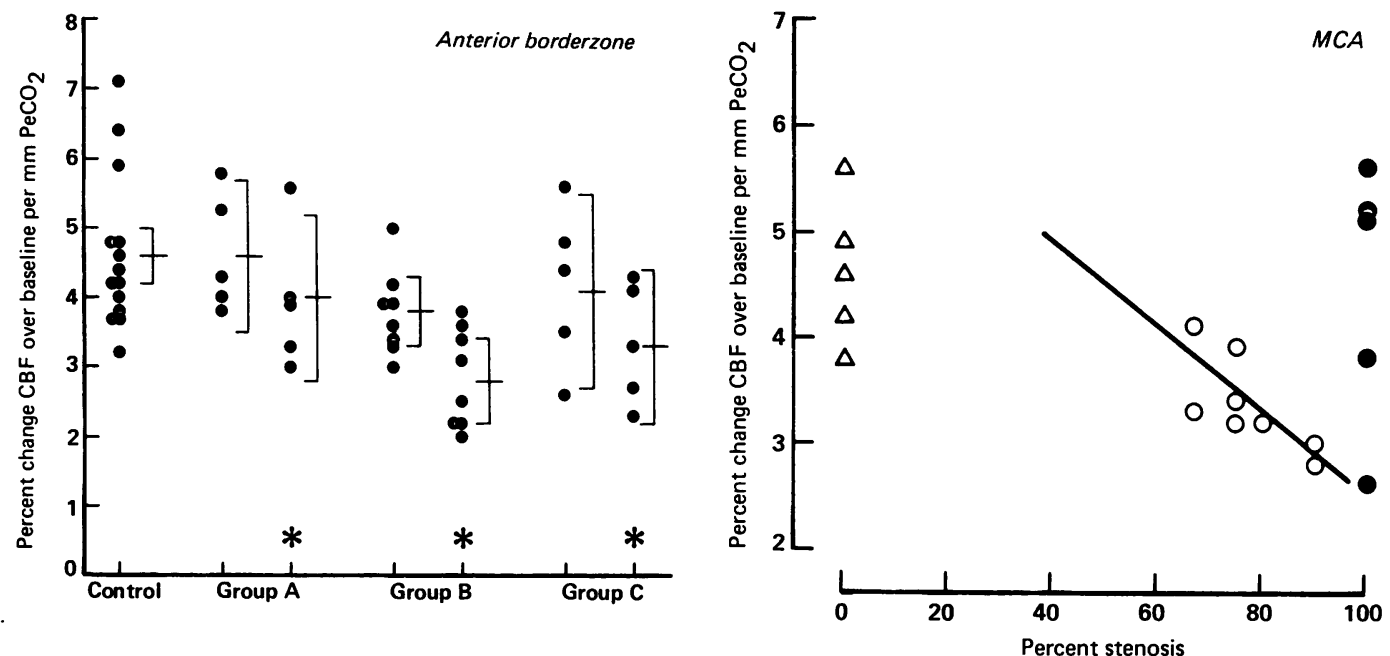

(fig 3) and statistically the ABZ RES\% of Group B was once again the lowest in comparisons made between patient groups ( $p<0.04$ ). Side to side ratios for all patient groups (fig 4) were significantly lower than controls $(p<0.04)$ but were not significantly different using between group comparisons. Symptomatic versus asymptomatic RES\% values were not significantly different within each respective group.

Blood flow detail-PBZ (table 2) While there were no statistically significant differences among PBZ values, RES\% of Group B patients were clearly the lowest [mean (SEM) 3.4 $(0 \cdot 2)$ ], symptomatic side) PBZ values and the most asymmetric. The Group B PBZ RES\% values approach significance $(0.25<p<0.10)$ using between group comparisons.

Arterial status (figs 5, 6) Linear regression showed significant relationships between symptomatic MCA RES\% of Group B and both percentage ICA stenosis $(p=0.04)$ and residual luminal diameter $(p=0.05)$. In spite of having the lowest mean (SEM) RES\%, 2.8 $(0.4)$, of all measurements, relationships between symptomatic ABZ RES\% of Group B

Figure 5 Linear regression of symptomatic $M C A$ $R E S \%$ versus percentage ICA stenosis. Group $B$ (open circle) data is expressed by the equation

$Y=6.5-(0.04) X\left(p=0.04, R^{2}=0.54\right)$ with Group $A$ (open triangle) and Group $C$ (filled circle) data depicted for comparison.

and both percentage ICA stenosis $(p=0.5)$ and residual luminal diameter $(p=0.3)$ only approached significance. No other significant relationships were evident.

Collateral patterns (table 4) Collateral patterns were not evident in Group A or B angiograms. The lowest MCA RES\% value in Group C corresponded to an ophthalmicreversal collateral pattern. The lowest ABZ RES\% value in Group C corresponded to a mixed ophthalmic/anterior Willisi collateral pattern. These were also the most asymmetric values.

\section{Discussion}

Between patient group comparisons most clearly delineated significant relationships between those patients with $50-99 \%$ unilateral carotid stenosis and CBF reactivity to induced hypercapnia. In this preliminary analysis, we
Figure 4 Individual $A B Z$ ratios for each subject with group means (SEM). Between patient group comparisons were unrevealing.

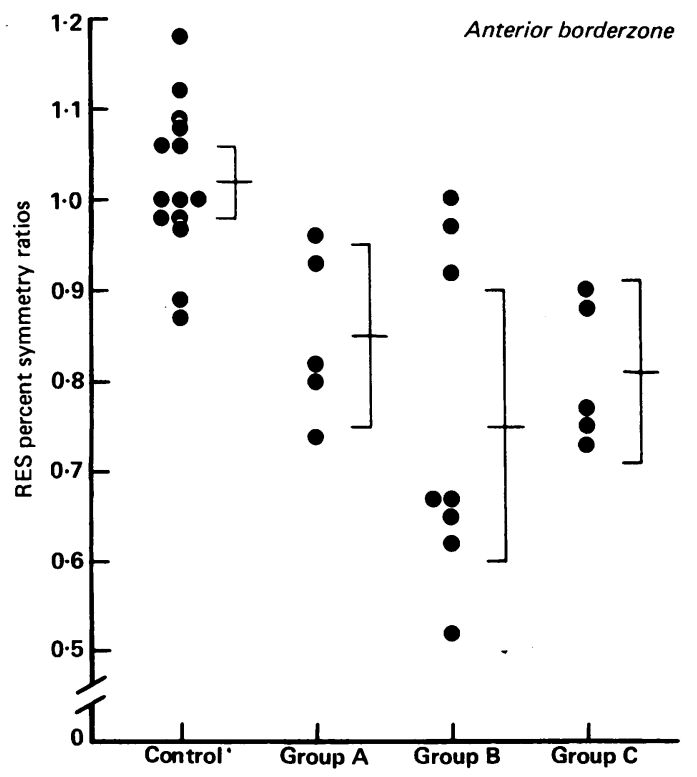

Figure 6 Linear regression of symptomatic $M C A$ $R E S \%$ versus residual luminal diameter. Group $B$ (open circle) data is expressed by the equation

$Y=(0.08) X+2.6\left(p=0.05, R^{2}=0.49\right)$ with Group $C$ (filled circle) data depicted for comparison. 
Table 4 Collateral patterns and reactivity ${ }^{\star}$

\begin{tabular}{lllll}
\hline Symptomatic & \multicolumn{5}{c}{ Collateral pattern } \\
\hline MCA RES\% & ABZ RES\% & Ophthalmic & Anterior & Posterior \\
$2 \cdot 6 \dagger$ & $2 \cdot 7$ & + & - & - \\
3.8 & $2 \cdot 3 \dagger$ & + & + & + \\
$5 \cdot 1$ & 3.3 & - & + & + \\
$5 \cdot 2$ & $4 \cdot 3$ & - & + & + \\
5.6 & $4 \cdot 1$ & & + & + \\
\hline
\end{tabular}

* Group C, ICA occlusion patients only

tOutside lower $95 \%$ confidence interval of normal subjects.

showed evidence of an inverse relationship between reactivity and residual luminal diameter. As our data set is expanded, we would expect to show a hyperbolic inverse relationship between absolute luminal crosssectional reductions and both CBF and RES\%. In addition, the bilateral reduction of reactivity in Group B patients suggests either a state of compensatory vasodilation of intracerebral arterioles or interhemispheric shunting of blood.

Normally, the addition of $\mathrm{CO}_{2}$ to the inhaled ${ }^{18} \mathrm{FCH}_{3}$ mixture is followed by intense cerebral vasodilation and increased CBF. The results of this study suggest that cerebral vasodilatory responses are significantly lowered in proportion to graded-arterial stenoses. We will, however, remain cautious in our conclusions regarding unilateral ICA stenosis and impaired cerebral vasocapacitance. We realise that our sample size is small and that alterations of vessel calibre are influenced by additional factors such as cerebrovascular resistance, turbulence of CBF, effects of endothelial damage and its contribution to thrombus formation. ${ }^{4}$

Although reduced $\mathrm{CO}_{2}$ responsivity can be a nonspecific finding, ${ }^{13-15}$ we report specific patterns of abnormal RES\% in patients with unilateral carotid disease, especially those with $50-99 \%$ stenosis, where age, $\mathrm{PeCO}_{2}, \mathrm{MABP}$, serum haematocrit and serum glucose values do not differ between groups. The relatively preserved $\mathrm{CO}_{2}$ responsivity in three of five ICA occlusion patients suggests that sufficient time may have elapsed to allow dilation of collateral pathways or that collateral anatomic pathways are present. ${ }^{1}$ If the collateral circulation is marginal, areas of potential ischaemia become maximally vasodilated and are thus unable to respond to the intense vasodilation promoted by induced hypercapnia. ${ }^{8}$ Our Group C patient with the lowest RES\% value had ophthalmic collaterisation, a pattern typically felt to carry a poor prognosis ${ }^{1617}$ and both continued and haemodynamically-induced symptomatology.

With respect to the reactivity of $\mathrm{CBF}$ to changes in $\mathrm{CO}_{2}$, Tsuda et al, using intraarterial Xenon, found reactivity preserved in both TIAs due to ICA occlusion and ICA mild stenosis. $^{18}$ They did not, however, study patients in the $50-99 \%$ stenosis group. Thompson, using an intravenous tracer clearance technique, found preserved $\mathrm{CO}_{2}$ reactivity in six TIA patients but did not relate CBF measurements to angiographic detail. ${ }^{19}$ In a study of patients with severe unilateral ICA stenosis $(75 \%)$ or occlusion, Keyeux et al, using Xenon inhalation, found that $\mathrm{CO}_{2}$ reactivity was substantially reduced in both hemispheres of all stenotic and occluded patients. ${ }^{20}$ They found that symptomatic hemispheres of ICA occlusion patients had the most abnormal RES\% of any group. Brown et al, using intravenous Xenon techniques, found that hemispheric $\mathrm{CO}_{2}$ reactivity was well-preserved in the majority of patients with unilateral carotid occlusion, but was significantly lower on the side of occlusion. ${ }^{21}$ They also found a close association between impaired $\mathrm{CO}_{2}$ reactivity and continuing TIAs. We have reported this previously. $^{8}$

In a study of patients with unilateral haemodynamically significant carotid artery disease ( $>66 \%$ diameter reduction), Powers et al, using oxygen-15 radiotracers, found a significant relationship between PET measurements and patterns of collateral circulation but not to measured reductions in luminal diameter. ${ }^{1}$ Our results, although obtained on a small sample size and with a lowresolution PET system, agree with those of Brown et al in the finding of a relationship of graduated reduction in reactivity with increasing severity of carotid artery disease. ${ }^{21}$ Final conclusions regarding our methodology and ICA occlusion awaits a larger study.

Our results would be even more compelling if matched cerebral blood volume (CBV)/CBF/ metabolic measurements could be done on those subjects who manifest abnormal $\mathrm{CO}_{2}$ reactivity, although Powers et al reported that there was no correlation between abnormal cerebral haemodynamic and stroke risk. ${ }^{22} \mathrm{We}$ would be especially interested in measurements of $\mathrm{CBV}$ in our patients, so that we could compare $\mathrm{CO}_{2}$ reactivity with $\mathrm{CBF} / \mathrm{CBV}$ ratios. Gibbs et al, using oxygen-15 and PET, reported that the $\mathrm{CBF} / \mathrm{CBV}$ ratios rose after extracranial-intracranial bypass surgery in each of 12 patients studied, ${ }^{23}$ while Halsley et al found that the most striking change after bypass surgery was a return towards normal $\mathrm{CO}_{2}$ activity. ${ }^{24}$

The predilection for the symptomatic $\mathrm{ABZ}$ territory in our patients, especially those with $50-99 \%$ stenosis, to have lowered $\mathrm{CO}_{2}$ reactivity parallels previous literature reports. ${ }^{122025}$ In a study of patients with predominantly unilateral severe $(>80 \%)$ carotid stenosis, Leblanc et al, using oxygen-15 and PET, found the ABZ to be selectively vulnerable. ${ }^{12}$ They demonstrated that cerebral haemodynamic function was abnormal in the $\mathrm{ABZ}$ ipsilateral to severe carotid stenosis, even when it was nor- 
mal in the MCA territory. Samson et al also observed diminished cerebral haemodynamics in MCA, ABZ, and PBZ territories in patients with either ICA or MCA occlusion. ${ }^{25}$ While $A B Z$ values were clearly the more asymmetric, symptomatic MCA values were the only territorial values that were significantly related to graded-arterial disease. Expansion of our data set should help clarify this relationship. Our finding of a symmetrical reduction of RES\% in both MCA territories, however, is difficult to understand because we also found a pronounced $\mathrm{ABZ}$ asymmetry.

We conclude that we can accurately and noninvasively evaluate the physiological effects of carotid artery disease on the ability of the cerebral circulation to respond to induced hypercapnia. Studies of $\mathrm{CO}_{2}$ reactivity illustrate both the efficiency of collateral circulatory patterns and, in a preliminary fashion, that moderate to severe, unilateral, symptomatic carotid arterial lesions impair cerebral vasocapacitance. Our findings need confirmation, however, relative to both newer generation, higher resolution PET systems currently available and longitudinal clinical follow up. ${ }^{22}$

The authors are indebted to Ms Sue Melvin and Ms Barb Rogers for preparing the manuscript, Ms Joan Hanson and Mr Bruce Rowe for their technical support and the Departments of Radiology and Nuclear Medicine for supporting this work.

1 Powers WJ, Press GA, Grubb RL, Gado M, Raichle ME. The effect of hemodynamically significant carotid artery disease on the hemodynamic status of the cerebral circulation. Ann Int Med 1987;106:27-35.

2 Grotta JC, Bigelow RH, Hu H, Fields WS. The significance of carotid stenosis or ulceration. Neurology 1984;34: $437-42$.

3 Javid H, Ostermiller WE, Hengesh JW, et al. Natural history of carotid bifurcation atheroma. Surgery 1970;67:80-6.

4 Grady PA. Pathophysiology of extracranial cerebral artery stenosis-A critical review. Stroke 1984;15:224-36.

5 DeWeese JA, May A, Lipchik EO, Rob CG. Anatomic and hemodynamic correlations in carotid artery stenosis. Stroke 1970;1:149-57.
6 Archie JP, Feldtman RW. Critical stenosis of the internal carotid artery. Surgery 1981;89:67-72.

7 Brice JG, Dowsett DJ, Lowe RD. Haemodynamic effects of carotid artery stenosis. BMJ 1964;2:1363-6.

8 Levine RL, Lagreze HL, Dobkin JA, et al. Cerebral vasocapacitance and TIA's. Neurology 1988;39:25-9.

9 Levine RL, Sunderland JJ, Lagreze HL, Nickles RJ, Rowe BR, Turski PA. Cerebral perfusion reserve indexes determined by fluoromethane positron emission scanning. Stroke 1988;19:19-27.

10 Olsen S, Larsen B, Herning M, Bech Skriver E, Lassen NA. Blood flow and vascular reactivity in collaterally perfused brain tissue. Evidence of ischemic penumbra in patients brain tissue. Evidence of ischemic penum

11 Von Schulthess GK, Ketz E, Schubiger PA, Bekier A. on Schulthess GK, Ketz E, Schubiger PA, Bekier A.
Regional quantitative noninvasive assessment of cerebral perfusion and function with $\mathrm{N}$-Isopropyl-Iodoamphetamine. J Nuc Med 1985;26:9-16.

12 Leblanc R, Yamamoto YL, Tyler JL, Diksic M, Hakim A. Borderzone ischemia. Ann Neurol 1987;22:707-13.

13 Grubb RL, Raichle ME, Eichling JO, Ter-Pogossian MM. The effects of changes in $\mathrm{PaCO}_{2}$ on cerebral blood volume, blood flow, and vascular mean transit time. Stroke 1974;5:630-9.

14 Nemoto EM, Snyder JV, Carroll RG, Morita H. Global ischemia in dogs: Cerebrovascular $\mathrm{CO}_{2}$ reactivity and autoregulation. Stroke 1975;6:425-31.

15 Heistad DD, Marcus ML, Piegors DJ, Armstrong ML. Regulation of cerebral blood flow in atherosclerotic monkeys. Am J Physiol 1980;239:H539-44.

16 Norrving B, Nilsson B, Cronquist S. Cerebral ischemic symptoms in carotid artery occlusion: Role of hemodynamic factors. Neurol Res 1981;3:125-38.

17 Norrving B, Nilsson B, Risber J. rCBF in patients with carotid occlusion. Resting and hypercapnic flow related to collateral pattern. Stroke 1982;13:155-62.

18 Tsuda Y, Kimura K, Yoneda S, et al. Cerebral blood flow and $\mathrm{CO}_{2}$ reactivity in transient ischaemic attacks: Comparison between TIA's due to the ICA occlusion and ICA mild stenosis. Neurol Res 1983;5:17-37.

19 Thompson SW. Reactivity of cerebral blood flow to $\mathrm{CO}_{2}$ in patients with transient cerebral ischemic attacks. Stroke patients with

20 Keyeux A, Laterre C, Becker C. Resting and hypercapnic rCBF in patients with unilateral occlusive disease of the rCBF in patients with unilateral occlusive disease of th

21 Brown MM, Wade JPH, Bishop CCR, Ross Russell RW. Reactivity of the cerebral circulation in patients with 49:899-904.

22 Powers WJ, Tempel LW, Grubb RL. Influence of cerebral hemodynamics on stroke risk: One-year follow-up of 30 medically treated patients. Ann Neurol 1989;25:325-30.

23 Gibbs JM, Wise RJS, Thomas DJ, Mansfield AO, RossRussell RW. Cerebral haemodynamic changes after extracranial-intracranial bypass surgery. $J$ Neurol Neurosurg Psychiatry 1987;50:140-50.

24 Halsley JH, Morawetz RB, Blaumenstein UW. The haemodynamic effect of STA/MCA bypass. Stroke 1982;13:163-7.

25 Samson Y, Baron JC, Bousser MG, et al. Effects of extraintracranial arterial bypass on cerebral blood flow and oxygen metabolism in humans. Stroke 1985;16:609-16. 